\title{
Linear response of vibrated granular systems to sudden changes in the vibration intensity
}

\author{
J. Javier Brey and A. Prados \\ Física Teórica, Universidad de Sevilla, Apartado de Correos 1065, 41080, Sevilla, Spain
}

(Received 15 January 2001; published 10 May 2001)

\begin{abstract}
The short-term memory effects recently observed in vibration-induced compaction of granular materials are studied. It is shown that they can be explained by means of quite plausible hypothesis about the mesoscopic description of the evolution of the system. The existence of a critical time separating regimes of "anomalous" and "normal" responses is predicted. A simple model fitting into the general framework is analyzed in the detail. The relationship between this paper and previous studies is discussed.
\end{abstract}

DOI: 10.1103/PhysRevE.63.061301

PACS number(s): 45.70.Cc, 61.43.Fs, 81.05.Rm

\section{INTRODUCTION}

Experiments have shown that when a loose packing of grains is submitted to vertical vibration or "tapping," it slowly approaches a steady state of higher packing fraction $[1,2]$. The final steady density is a decreasing function of the dimensionless parameter characterizing the vibration intensity. Moreover, the relaxation is slower for smaller vibration intensity. In the time evolution of the system neither convection effects nor oscillatory behavior are observed. The study of the kinetics of compaction is important both from a formal point of view and because of its economical relevance in many industrial processes. Most of the peculiar behaviors exhibited by granular materials submitted to vibration or tapping processes show a great similarity with conventional structural glasses. This includes slow relaxation, annealing properties, and hysteresis effects.

The first study of the response of a granular system to a sudden change in the vibration intensity we are aware of, was carried out by means of numerical simulations of a model for compaction [3], and the data indicated the presence of memory effects in the evolution of the density of the system. Very recently [4], memory effects were also directly observed in a series of experiments. The results showed that the system has a short-term memory of its shaking history, so that the response in the evolution of the density to a change in the vibration intensity at a given time, is not determined by the density at that time. Mathematically, this phenomenon implies that the time evolution of the density does not obey a closed ordinary first-order differential equation.

In this paper, we propose a general theoretical framework to understand the origin and characteristic features of the memory effects seen both in simulations and in experiments. Using quite plausible hypothesis, we will be able to explain the short-time response of the system to a small change in the vibration intensity. In particular, the theory predicts that a decrease (increase) in the intensity can lead to an increase (decrease) of the compaction rate on short-time scales, in agreement with experiments. Nevertheless, is not necessarily so. If the change in the intensity is made at the early stages of the compaction process, the theory we will develop leads to a modification of the compaction rate having the same sign as the intensity change. In fact, there is a critical time, which depends on the tapping intensity before the change, separating the regions of "normal" and "anomalous" responses.
The existence of these two different regimes has not been verified experimentally up to now, although such a behavior has been numerically observed in a simple model for granular compaction [5].

As an illustration of the theory, we discuss its application to a model for compaction introduced recently [6,7]. The model has already been shown to reproduce the qualitative behavior of granular materials under tapping. Here we will show that it also captures the same short-term memory effects seen in the experiments. Moreover, it fits perfectly in the general scheme developed in this paper, therefore providing a first test of validation of the ideas in which the theory is based. We have also used the model to investigate the relaxation of the system following a perturbation in the vibration intensity for a short-time period. This idea also originates from the experiments reported in Ref. [4]. The results indicate that the response function is accurately described by a Kohlrausch-Williams-Watts (KWW) or stretched exponential function.

The paper is organized as follows. In the next section, some general properties of the equation governing the time evolution of the density in tapping processes of granular media are discussed. These properties are used in Sec. III to analyze the short-term memory effects by considering the response of the system to a small change in the vibration intensity. The theory is particularized for a simple model for tapping in Sec. IV, where other patterns of change of the vibration intensity are also considered. The choices were originated from the experiments reported in Ref. [4]. Finally, Sec. V contains some additional comments and final remarks, as well as a relevant discussion of the relationship of our paper to previous experimental and theoretical studies.

\section{EVOLUTION OF THE DENSITY IN DISCRETE TAPPING PROCESSES}

Let $\Gamma$ denote the dimensionless parameter characterizing the intensity of the vibration applied to the granular medium. In typical experiments [1], $\Gamma$ is defined as the ratio of peak acceleration of a tap to the gravity $g$. Under very general conditions, the time evolution of the density $\rho$ in a discrete tapping process will be given at a mesoscopic level by an equation of the form 


$$
\dot{\rho} \equiv \frac{d \rho(t)}{d t}=f_{1}(\Gamma) \mu_{1}(t)-f_{2}(\Gamma) \mu_{2}(t)
$$

Here the time is measured in units of complete taps in some continuous limit, $f_{1}(\Gamma)$ and $f_{2}(\Gamma)$ are semidefinite positive functions of $\Gamma$ having dimensions of frequency, and $\mu_{1}(t)$ and $\mu_{2}(t)$ are positive quantities depending on the state of the system, but they are not univocally determined by the density at the same instant $\rho(t)$. Therefore, Eq. (1) is not in general a closed equation and cannot be solved by itself. The two terms on the right-hand side of the equation describe elementary processes increasing and decreasing the density, respectively.

The structure of Eq. (1) as a gain-loss equation is consistent with the experimental observations in compaction processes, as we will discuss in detail in the following. Also, if the elementary events taking place in the system being vibrated can be described by means of a Master equation, a formal equation like this follows directly. This is the case for some simple kinetic models for compaction introduced recently [6-9].

Since Eq. (1) describes the evolution of the density as a consequence of tapping, the functions $f_{1}$ and $f_{2}$ must vanish in the limit of no tapping $\Gamma=0$, so that

$$
f_{1}(0)=f_{2}(0)=0 \text {. }
$$

Because of continuity, it follows, at least for small values of the intensity $\Gamma$, that

$$
f_{1}^{\prime}(\Gamma) \equiv \frac{d}{d \Gamma} f_{1}(\Gamma)>0, \quad f_{2}^{\prime}(\Gamma) \equiv \frac{d}{d \Gamma} f_{2}(\Gamma)>0 .
$$

We will assume that the above inequalities hold for arbitrary $\Gamma$. The physical reason for this assumption is that we expect the number of elementary processes taking place in the system to increase as $\Gamma$ increases. Of course, this does not imply by itself that the rate of variation of the density also increases. The behavior of $\dot{\rho}$ depends on the net balance between the gain and loss elementary events, as indicated by Eq. (1). This picture is in agreement with the qualitative role of temperature played by the shaking intensity in many different aspects $[2,7,10-13]$.

In the long-time limit of a tapping process with constant $\Gamma$, the experiments show that the system reaches a steady state with a density $\rho_{s}$, which is a monotonic decreasing function of $\Gamma$, as displayed by the "reversible" branch in cycling experiments [9]. Let us point out that the relaxation process is very slow, and for very small values of $\Gamma$ the steady density is hard to reach within the experimental time scale. Therefore, the function $\rho_{s}(\Gamma)$ verifies that $d \rho_{s}(\Gamma) / d \Gamma<0$, and it is bounded by the two formal limits

$$
\rho_{\min }=\lim _{\Gamma \rightarrow \infty} \rho_{s}(\Gamma), \quad \rho_{\max }=\lim _{\Gamma \rightarrow 0} \rho_{s}(\Gamma) .
$$

Particularization of Eq. (1) for a steady state yields

$$
f_{1}(\Gamma) \mu_{1 s}=f_{2}(\Gamma) \mu_{2 s},
$$

where $\mu_{1 s}$ and $\mu_{2 s}$ denote the steady values of the quantities $\mu_{1}$ and $\mu_{2}$, respectively. As pointed out above, $\mu_{1}(t)$ and $\mu_{2}(t)$ are not expected to be simply functions of $\rho(t)$ in general. But, on the other hand, it seems sensible to assume that the steady state reached by a given system in a tapping experiment is fully determined by the intensity $\Gamma$ or, equivalently, by $\rho_{s}$. Therefore, we assume that $\mu_{1 s}$ and $\mu_{2 s}$ are functions of $\rho_{s}$, and in the following we are going to investigate some qualitative properties of these functions. For $\rho_{s}$ $\rightarrow \rho_{\min }, \mu_{2 s}\left(\rho_{s}\right)$ must vanish, since by definition at $\rho_{s}$ $=\rho_{\min }$ there are no processes decreasing the density. Therefore, it is

$$
\lim _{\rho_{s} \rightarrow \rho_{\min }} \mu_{2 s}\left(\rho_{s}\right)=0, \quad \lim _{\rho_{s} \rightarrow \rho_{\min }} \mu_{1 s}\left(\rho_{s}\right)>0 .
$$

The second relationship expresses that starting from the density $\rho_{\text {min }}$, any tapping process of arbitrary intensity $\Gamma$ can only produce an increase of the density. What happens in the steady high-density limit? A similar argument to the one carried out above would lead to

$$
\lim _{\rho_{s} \rightarrow \rho_{\max }} \mu_{1 s}\left(\rho_{s}\right)=0, \quad \lim _{\rho_{s} \rightarrow \rho_{\max }} \mu_{2 s}\left(\rho_{s}\right)>0 .
$$

Nevertheless, some care is required when analyzing this limit. Simple models for discrete tapping lead to an absorbent steady state in the high-density limit $[6,7]$. That means that the system will not be able to leave this state when submitted to tapping of arbitrary intensity. This is equivalent to saying that $\mu_{2 s}\left(\rho_{s}\right)$ also vanishes for $\rho_{s} \rightarrow \rho_{\max }$. As a consequence, and in order to include such a possibility in our formulation, instead of Eq. (7) we will assume the more general and precise condition

$$
\lim _{\rho_{s} \rightarrow \rho_{\max }} \frac{\mu_{1 s}\left(\rho_{s}\right)}{\mu_{2 s}\left(\rho_{s}\right)}=0
$$

i.e., $\mu_{2 s} \gg \mu_{1 s}$ when $\rho_{s} \rightarrow \rho_{\max }$, and the density loss term is dominant in that limit. Let us note that Eq. (6) yield

$$
\lim _{\rho_{s} \rightarrow \rho_{\text {min }}} \frac{\mu_{1 s}\left(\rho_{s}\right)}{\mu_{2 s}\left(\rho_{s}\right)}=\infty .
$$

The simplest behavior that is consistent with Eqs. (8) and (9) is that the ratio $\mu_{1 s} / \mu_{2 s}$ be a monotonic decreasing function of the steady density $\rho_{s}$ going from infinity to zero. Since there is not any physical reason to expect a more complicated density dependence, we assume this is the case in our formalism. From the steady condition given by Eq. (5), it follows that

$$
\frac{\mu_{1 s}}{\mu_{2 s}}=\frac{f_{2}(\Gamma)}{f_{1}(\Gamma)} \equiv g(\Gamma) .
$$

The function $g(\Gamma)$ is a measure of the rate of the decompaction processes with respect to the rate of the compaction ones. Because of Eqs. (8) and (9), it is 


$$
\lim _{\Gamma \rightarrow \infty} g(\Gamma)=\infty, \quad \lim _{\Gamma \rightarrow 0} g(\Gamma)=0 .
$$

Taking the derivative with respect to the intensity $\Gamma$ in Eq. (10), we obtain:

$$
\frac{d g(\Gamma)}{d \Gamma}=\frac{d \rho_{s}}{d \Gamma} \frac{d}{d \rho_{s}}\left(\frac{\mu_{1 s}}{\mu_{2 s}}\right)>0,
$$

where we have taken into account the monotonically decreasing density dependence of $\mu_{1 s} / \mu_{2 s}$ assumed above. The physical meaning of Eq. (12) is evident; the rate of the decompaction processes grows faster with $\Gamma$ than the rate of the compaction processes. Also this implication of our assumptions seems physically plausible.

In summary, we can write the equation for the time evolution of the density in discrete tapping processes as

$$
\frac{d \rho(t)}{d t}=f_{1}(\Gamma)\left[\mu_{1}(t)-g(\Gamma) \mu_{2}(t)\right]
$$

with $f_{1}$ and $g$ being positive increasing functions of $\Gamma$, both of them vanishing in the limit $\Gamma \rightarrow 0$. The quantities $\mu_{1}(t)$ and $\mu_{2}(t)$ are some moments of the complete distribution function of the system, and they contain the influence of correlations on the evolution of the density. As a consequence, Eq. (13) is not a closed equation.

Because $g(\Gamma)$ vanishes for $\Gamma \rightarrow 0$, if a tapping experiment with low enough intensity $\Gamma$ is carried out, the decompaction term $f_{1}(\Gamma) g(\Gamma) \mu_{2}(t)$ will be negligible in the first stages of the process, i.e.,

$$
\frac{\mu_{1}(t)}{g(\Gamma) \mu_{2}(t)} \gg 1,
$$

and the evolution of the system will be approximately described by

$$
\frac{d \rho(t)}{d t} \simeq f_{1}(\Gamma) \mu_{1}(t) .
$$

At much later times, when $\rho(t)$ is close enough to the asymptotic steady value, the decompaction contribution in Eq. (13) plays a decisive role, leading to a steady density $\rho_{s}<\rho_{\text {max }}$, and it is

$$
\frac{\mu_{1}(t)}{g(\Gamma) \mu_{2}(t)}=O(1) .
$$

The observed behavior that the system tends towards a steady state and, therefore, a regime where $\mu_{1}, \mu_{2}$, and $g$ verify a relationship of the form given in Eq. (16), can be understood if $\mu_{1}(t)$ decreases in time while $\mu_{2}$ increases. Quite interestingly, this is consistent with a mean-field approximation in which $\mu_{1}(t)$ is replaced by $\mu_{1 s}[\rho(t)]$ and $\mu_{2}(t)$ by $\mu_{2 s}[\rho(t)]$. Since $\rho(t)$ increases monotonically in time, and $\mu_{1 s} / \mu_{2 s}$ is a monotonic decreasing function of the density, it follows that the left-hand side of Eq. (16) will decay in time.
Of course, as long as Eq. (15) is accurate, the larger the $\Gamma$ the faster the compaction of the system, in agreement with experiments. Over a larger time scale, the complete Eq. (15), including the decompaction term, is needed in order to explain the dependence of the steady density $\rho_{s}$ on $\Gamma$, and also the existence of a a slow long-time tail in the relaxation of the density, once $\rho_{s}-\rho(t)$ is very small. In this context, the presence of an "anomalous" density relaxation, following an inverse logarithm law, would be associated to some specific dynamical properties of the compaction term $\mu_{1}$ when the system is submitted to "nonlinear" tapping processes $[1,2]$. We use the term "nonlinear' here in the sense that, in the experiments, the initial value of the density is not very close to the steady density.

Later on, we will show that an evolution equation like Eq. (13) applies in the case of a simple model recently introduced to describe discrete tapping $[6,7,10]$. Another similar equation is obtained for the "parking' model $[8,9,14,15]$, although this latter refers to continuous vibration processes, in which the system is not allowed to relax to a metastable configuration between every two vibration cycles. In the parking model, the state of maximum density $\rho_{\max }$ is not totally absorbent, but this possibility has been included in our theory, as discussed below Eq. (7). By identifying the intensity of tapping $\Gamma$ with the ratio between the desorption and adsorption rates in the parking model, it is trivial to check by using the expressions in Ref. [8] that the quantities corresponding to $\mu_{1 s}$ and $\mu_{2 s}$ verify that their ratio is an increasing function of $\rho_{s}$, and also the limiting behavior given in Eqs. (8) and (9). Moreover, the steady density is an increasing function of the quantity playing the role of the vibration intensity. In conclusion, the parking model belong to the general class of systems we have considered.

\section{RESPONSE TO SMALL VIBRATION INTENSITY JUMPS}

In this section we will investigate whether Eq. (13), which has been built under very general arguments and is expected to have a wide range of applicability, is able to predict the memory effects recently observed in vibration-induced compaction in granular materials [4]. The fact that the equation is not closed for the density, implies that its time evolution in a given experiment with constant $\Gamma$ is not determined by its the initial value. Starting from the same value $\rho_{0}$, different time evolutions are possible depending on the way in which the system was prepared. Our aim is to analyze some particular relevant manifestations of this general statement.

Consider that, starting from a given configuration, the system is tapped with an intensity $\Gamma$. At a certain time $t_{w}$, the intensity is instantaneously changed to $\Gamma+\Delta \Gamma$. Quite peculiarly, it has been observed in the experiments that the change in the compaction rate has opposite sign that of $\Delta \Gamma$ on short time scales, though in the long-time regime the relaxation is slower for smaller values of the intensity of vibration $\Gamma$. The same kind of effect has also been previously found numerically in some models for compaction $[5,16,3]$, although it only shows up when the time interval $t_{w}$ is not too short. If $\Gamma$ is changed at the beginning of the compaction 
process, the variation of the compaction rate has the same sign as $\Delta \Gamma[5,17]$.

Application of Eq. (13) for the instant $t_{w}^{-}$, just before the change in the intensity of the vibration, yields

$$
r_{w} \equiv \dot{\rho}\left(t_{w}^{-}\right)=f_{1}(\Gamma)\left[\mu_{1 w}^{-}-g(\Gamma) \mu_{2 w}^{-}\right],
$$

where $\mu_{1 w}^{-}=\mu_{1}\left(t_{w}^{-}\right)$and $\mu_{2 w}^{-}=\mu_{2}\left(t_{w}^{-}\right)$. When the intensity of vibration is changed into $\Gamma+\Delta \Gamma$, the compaction rate becomes

$$
r_{w}^{\prime} \equiv \dot{\rho}\left(t_{w}^{+}\right)=f_{1}(\Gamma+\Delta \Gamma)\left[\mu_{1 w}^{+}-g(\Gamma+\Delta \Gamma) \mu_{2 w}^{+}\right] .
$$

The continuity of the distribution function of the system implies that $\mu_{1}^{-}=\mu_{1}^{+}$and $\mu_{2}^{-}=\mu_{2}^{+}$for an instantaneous jump of $\Gamma$, although there is a discontinuity $\Delta r_{w}=r_{w}^{\prime}-r_{w}$ in the compaction rate. For $\Delta \Gamma$ small we can approximate

$$
\begin{aligned}
\frac{\Delta r_{w}}{\Delta \Gamma}= & f_{1}^{\prime}(\Gamma)\left[\mu_{1 w}-g(\Gamma) \mu_{2 w}\right]-f_{1}(\Gamma) g^{\prime}(\Gamma) \mu_{2 w}=\frac{f_{1}^{\prime}(\Gamma)}{f_{1}(\Gamma)} r_{w} \\
& -f_{1}(\Gamma) g^{\prime}(\Gamma) \mu_{2 w} .
\end{aligned}
$$

Therefore, if over the compaction curve corresponding to intensity $\Gamma$, we define the function

$$
\lambda(t)=\frac{f_{1}^{\prime}(\Gamma)}{f_{1}(\Gamma)} r(t)-f_{1}(\Gamma) g^{\prime}(\Gamma) \mu_{2}(t),
$$

the sign of this function at the time $t_{w}$ when the intensity is changed will determine the relative behavior of $\Delta r_{w}$ with respect to $\Delta \Gamma$, for infinitesimal changes of the latter. If $\lambda_{w}$ $\equiv \lambda\left(t_{w}\right)<0$, the anomalous response observed in the experiments will follow, while if $\lambda_{w}>0$ the compaction rate will change in the same direction as $\Delta \Gamma$. Let us analyze the sign of the function $\lambda(t)$. In the long-time limit, formally $t_{w}$ $\rightarrow \infty$, the system is known to reach the asymptotic steady density, so that $r_{w} \rightarrow 0$ and, consequently,

$$
\lambda_{\infty}=\lim _{t \rightarrow \infty} \lambda(t)=-f_{1}(\Gamma) g^{\prime}(\Gamma) \mu_{2 s}(\Gamma)<0,
$$

where it has been taken into account that both $f_{1}(\Gamma)$ and $g_{1}(\Gamma)$ are positive increasing functions of $\Gamma$ and that $\mu_{2 s}(\Gamma)>0$.

On the other hand, if the initial density in the experiment is the minimum possible density of the system at rest $\rho_{\text {min }}$, corresponding to the random loose packing configuration, it follows from the properties of $\mu_{2}$ that

$$
\lim _{t \rightarrow 0} \lambda(t)=\frac{f_{1}^{\prime}(\Gamma)}{f_{1}(\Gamma)} r(0)>0
$$

Even though we have considered in our discussion that $\rho(t$ $=0)=\rho_{\text {min }}$ in order to derive the above inequality, the same result will apply if the initial density is close enough to it, so that the first term on the right-hand side of Eq. (20) dominates the second one in the initial regime.

Then, we conclude that for short times, $\Delta r_{w}$ and $\Delta \Gamma$ have the same sign, while for large times their signs are opposite.
This renders compatible and explains what is seen in the experiments and also in numerical studies of simple models. From our analysis it follows that there is (at least) a time $t_{c}$, which depends on the value of $\Gamma$, such that the response of the system to a small variation of the intensity of tapping is qualitatively different for $t<t_{c}$ and $t>t_{c}$.

The study carried out in this section has been restricted to small instantaneous changes in $\Gamma$, allowing the use of a linear analysis of Eq. (13). Whether the behavior of the system remains the same when submitted to a finite change in the shaking intensity, it cannot be inferred from our analysis. In this case, nonlinear effects can modify dramatically the response of the system. More will be said about this in the next section of the paper.

\section{APPLICATION TO A SIMPLE MODEL FOR COMPACTION}

The general scenario developed in the previous sections will be particularized here for a one-dimensional lattice model for compaction $[7,10]$. In the model, each site $i$ can be either empty or occupied by a particle. A variable $m_{i}$ is defined, being $m_{i}=1$ in the former case and $m_{i}=0$ in the latter. A configuration of the system is fully specified by giving the values of all the variables $\mathbf{m} \equiv\left\{m_{i}\right\}$. As usual, we will refer to the empty sites as being occupied by a hole.

Let us describe the dynamics of the system when submitted to a discrete tapping process. Mechanical stability requires that all the holes be isolated, i.e., surrounded by two particles, at the end of every tap. The time evolution of the system is defined as a Markov process, and formulated by means of a Master equation for the probability distribution of the system $[7,10]$. The equation contains the transition rates $W\left(\mathbf{m} \mid \mathbf{m}^{\prime}\right)$ from state $\mathbf{m}^{\prime}$ to state $\mathbf{m}$. There are three kinds of possible transitions. Indicating only the variables associated to the sites involved in the transitions, the nonvanishing transition rates are

(1) Elementary diffusive events conserving the number of particles,

$$
W(010 \mid 100)=W(010 \mid 001)=\frac{\alpha}{2},
$$

(2) Transitions increasing the number of particles,

$$
\begin{gathered}
W(010 \mid 101)=\frac{\alpha}{2}, \\
W(001 \mid 101)=W(100 \mid 101)=\frac{\alpha}{4} .
\end{gathered}
$$

(3) Transitions increasing the number of holes, i.e., decreasing the number of particles,

$$
\begin{gathered}
W(01010 \mid 00100)=\frac{\alpha^{2}}{2}, \\
W(01010 \mid 01000)=W(01010 \mid 00010)=\frac{\alpha^{2}}{4} .
\end{gathered}
$$


In the above equations, $\alpha$ is a positive constant, characterizing the tapping process completely, and playing in the model a role similar to the intensity of vibration $\Gamma$ in real experiments. For $\alpha \neq 0$, the system evolves from any arbitrary initial configuration to a final steady state with density

$$
\rho_{s}(\alpha)=\frac{1}{2}\left[1+(1+4 \alpha)^{-1 / 2}\right] .
$$

From here it follows that

$$
\lim _{\alpha \rightarrow 0} \rho_{s}=1 \equiv \rho_{\max }, \quad \lim _{\alpha \rightarrow \infty} \rho_{s}=\frac{1}{2} \equiv \rho_{\min },
$$

being

$$
\frac{d \rho_{s}}{d \alpha}<0
$$

for all $\alpha$. Therefore the density in the model has the same kind of dependence on the intensity $\alpha$ as assumed in the general discussion in Sec. II. The time evolution of $\rho$ is obtained from the Master equation for the model, and reads [18]

$$
\dot{\rho}=\alpha x_{101}(t)-\frac{\alpha^{2}}{2}\left[x_{00100}(t)+\frac{1}{2} x_{01000}(t)+\frac{1}{2} x_{00010}(t)\right],
$$

where $x_{010}$ is the concentration of three-site clusters of the form hole-particle-hole, $x_{00100}$ is the concentration of fivesite clusters formed by a hole between two pairs of particles, and so on. Comparison of Eqs. (1) and (29) allows to identify

$$
\begin{gathered}
f_{1}(\alpha)=\alpha, \quad f_{2}(\alpha)=\alpha^{2}, \\
\mu_{1}(t)=x_{101}(t) \\
\mu_{2}(t)=\frac{1}{2} x_{00100}(t)+\frac{1}{4}\left[x_{01000}(t)+x_{00010}(t)\right] .
\end{gathered}
$$

In the steady state, the only correlations in the system are those forbidding to have two nearest-neighbor holes [18]. Then, it is a simple matter to compute the steady values of the several cluster concentrations appearing in Eq. (31) with the result

$$
\begin{gathered}
\mu_{1 s}=\frac{\left(1-\rho_{s}\right)^{2}}{\rho_{s}}, \\
\mu_{2 s}=\frac{\left(1-\rho_{s}\right)\left(2 \rho_{s}-1\right)^{2}}{\rho_{s}^{2}} .
\end{gathered}
$$

In the limit $\rho_{s} \rightarrow \rho_{\min }=1 / 2$,

$$
\mu_{1 s} \rightarrow \frac{1}{2}, \quad \mu_{2 s} \rightarrow 0,
$$

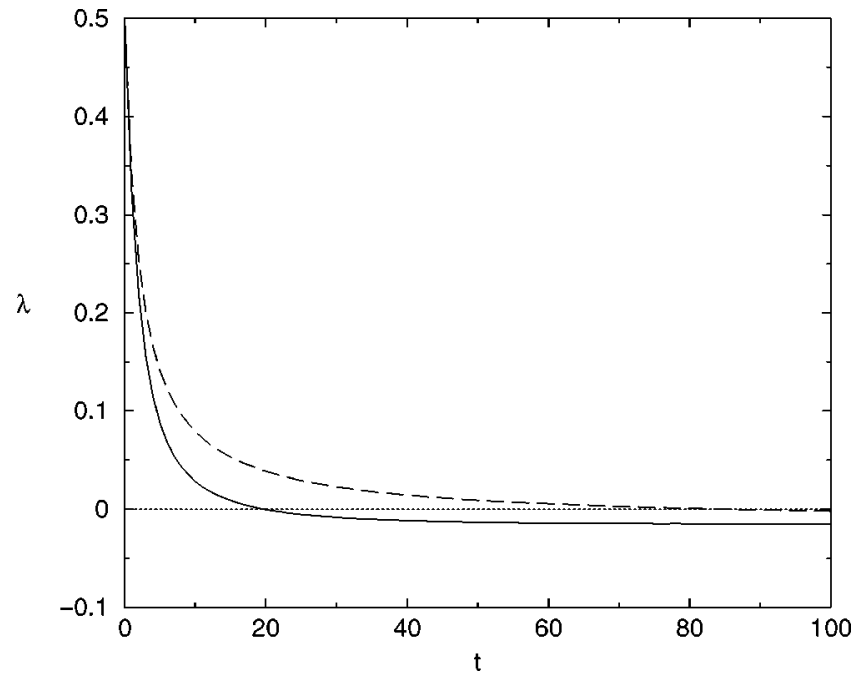

FIG. 1. Time evolution of the function $\lambda$ defined in Eq. (39), for a vibration intensity $\alpha=0.15$ (solid line). Also plotted is the meanfield approximation for $\lambda$ (dashed line).

while in the high-density limit $\rho_{s} \rightarrow \rho_{\max }=1$, both $\mu_{1 s}$ and $\mu_{2 s}$ vanish, as a consequence of the absorbent character of the state with all the sites occupied by particles. The ratio

$$
\frac{\mu_{1 s}\left(\rho_{s}\right)}{\mu_{2 s}\left(\rho_{s}\right)}=\frac{\rho_{s}\left(\rho_{s}-1\right)}{\left(2 \rho_{s}-1\right)^{2}}
$$

vanishes in this latter limit. Equations (34) and (35) are in agreement with Eqs. (6) and (8). Moreover, $\mu_{1 s}\left(\rho_{s}\right) / \mu_{2 s}\left(\rho_{s}\right)$ is a monotonic decreasing function of $\rho_{s}$ and, consistently [see Eq. (10)],

$$
g(\alpha)=\frac{f_{2}(\alpha)}{f_{1}(\alpha)}=\alpha
$$

is an increasing function of $\alpha$, vanishing in the limit $\alpha \rightarrow 0$.

We conclude that this model for compaction fits perfectly the general picture developed in the previous sections. Equation (13) particularized for the model is

$$
\frac{d \rho(t)}{d t}=\alpha\left(\mu_{1}-\alpha \mu_{2}\right),
$$

with $\mu_{1}$ and $\mu_{2}$ defined in Eq. (31). To solve Eq. (37) we would need some (approximate) expressions for the cluster concentrations as functions of the density.

If we submit the system to the tapping experiment described in Sec. III, the effect of the intensity change $\Delta \alpha$ at $t=t_{w}$ on the compact rate will be given by

$$
\frac{\Delta r_{w}}{\Delta \alpha}=\frac{r_{w}}{\alpha}-\alpha \mu_{2 w},
$$

in the limit of small $\Delta \alpha$. Therefore, the function determining whether the response of the system will be "normal" or "anomalous" is 


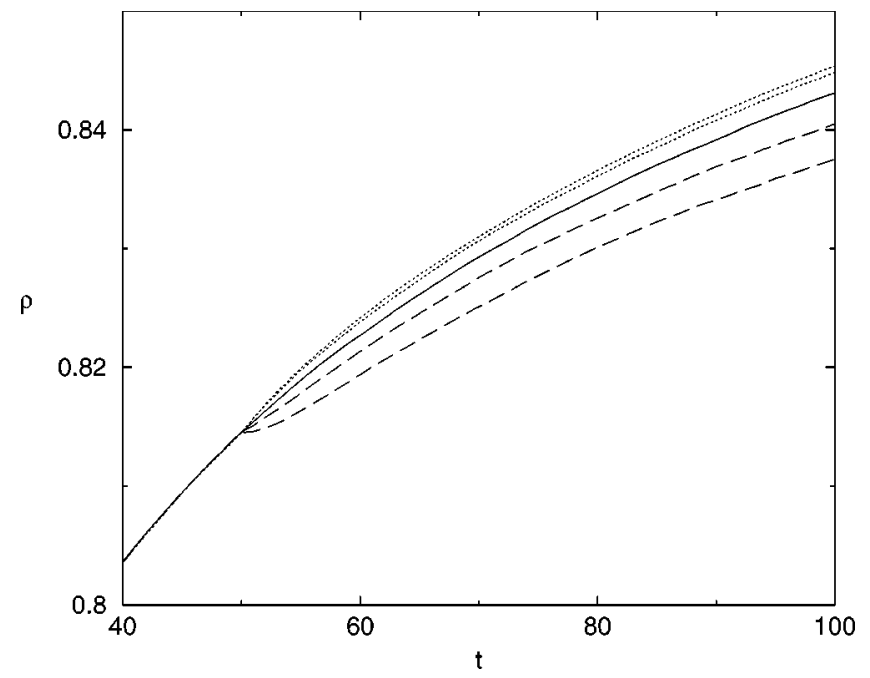

FIG. 2. Evolution of the density $\rho$, as a function of the number of taps $t$. Five numerical experiments are shown, the vibration intensity $\alpha$ was changed at $t_{w}=50$, where $\lambda<0$, from 0.15 to 0.1 , $0.125,0.15,0.175$, and 0.2 , from top to bottom. Thus, the central curve corresponds to no change in the tapping intensity $\alpha$ (solid line), while the dotted and dashed lines correspond to a decrease and an increase in $\alpha$, respectively. The "anomalous" response experimentally observed shows up.

$$
\lambda(t)=\frac{r(t)}{\alpha}-\alpha \mu_{2}(t) .
$$

In Fig. 1 this function is plotted for $\alpha=0.15$. The curve has been obtained by Monte Carlo simulation of the Master equation of the system. The data represent an average over 10 different runs. The initial state was the one corresponding to the steady minimum density. For this particular value of the intensity $\alpha, \lambda(t)$ changes sign between taps 19 and 20, i.e., $19 \leqslant t_{c} \leqslant 20$. For comparison purposes, we have also plotted the mean-field approximation for $\lambda(t)$ (dashed line). The latter has been constructed by substituting in Eq. (39) $\mu_{1}(t)$ and $\mu_{2}(t)$ by $\mu_{1 s}[\rho(t)]$ and $\mu_{2 s}[\rho(t)]$, respectively, and using for the density the simulation results. It is seen that the mean-field approximation also changes sign, but for larger times, and it is always above the "exact", Monte Carlo curve. This is consistent with the mean-field approximation giving a faster approach to the steady state than the actual relaxation of the system [18].

According to the results derived in this paper, $\Delta r_{w}$ is expected to have a different sign than that $\Delta \alpha$ for $t_{w}>t_{c}$ and the same for $t_{w}<t_{c}$. In order to check this theoretical prediction, we have carried out series of Monte Carlo simulations, all of them starting in the minimum density configuration, with $\alpha=0.15$. At $t_{w}=50$, the value of the intensity $\alpha$ was instantaneously changed to $\alpha^{\prime}$. The results for four different values of $\alpha^{\prime}$ are reported in Fig. 2, namely, $0.1,0.125,0.15,0.175$, and 0.2 , from top to bottom. The central value corresponds to no change. Since in these simulations it is $t_{w}>t_{c}$, the compaction rate is observed to decrease as the value of $\alpha^{\prime}$ increases. It is also seen that the amplitude of the jump in the compaction rate is larger for $\alpha^{\prime}=0.2$

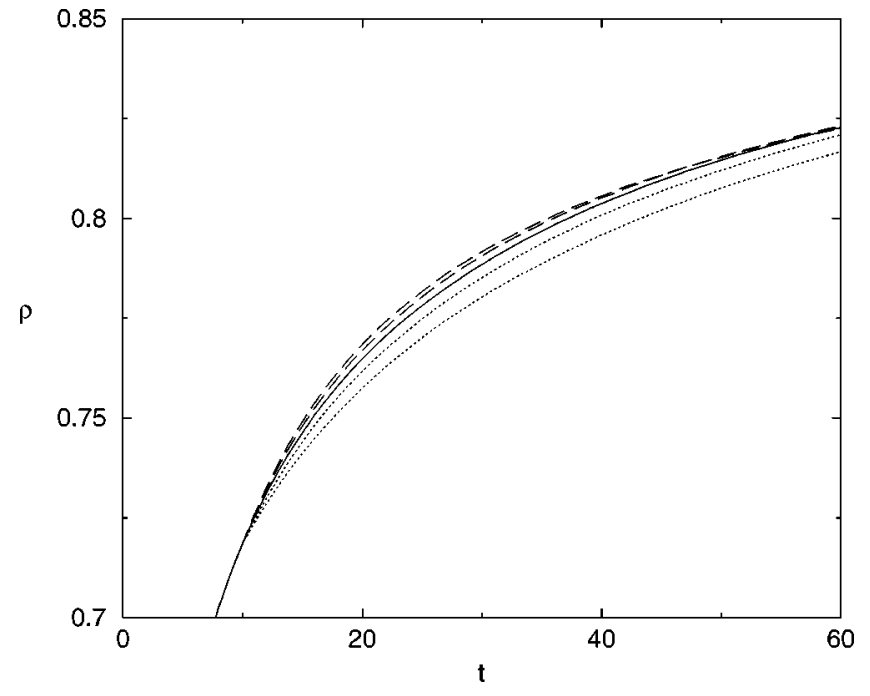

FIG. 3. The same experiment of Fig. 2, but the change in intensity is introduced at an earlier time $t_{w}=10$, at which $\lambda>0$. The curves correspond to the same values of $\alpha^{\prime}$ as in Fig. 2, but now are ordered from bottom to top. In this region the response is "normal," i.e., the compaction rate increases with the vibration intensity.

( $\Delta \alpha=0.05)$ than for $\alpha^{\prime}=0.1(\Delta \alpha=-0.05)$. This feature cannot be explained by Eq. (38), and it is due to nonlinear effects that have been neglected in the linear approximation used here. This will be analyzed below.

In Fig. 3 the same set of experiments is carried out, with the only difference that in this case the intensity $\alpha$ is modified at $t_{w}=10<t_{c}$. The several curves correspond to the same values as in Fig. 2, but now they are ordered from bottom to top. As predicted by the theory, the variation of the compaction rate has the same sign as the change in $\alpha$. Moreover, the same kind of nonlinear effects as in Fig. 2 are present.

Now, we will briefly discuss the nonlinear corrections in $\Delta \alpha$ to the change in the compaction rate. It is easy to show that

$$
\Delta r_{w}=\Delta \alpha \lambda_{w}-(\Delta \alpha)^{2} \mu_{2 w}
$$

The second term on the right-hand side of Eq. (40) is neglected in the linear approximation. In this simple model, the nonlinear correction is always negative, so it can modify dramatically the response of the system to the jump $\Delta \alpha$ if the linear term $\Delta \alpha \lambda_{w}>0$. In particular, there is a critical value

$$
\Delta \alpha_{c}=\frac{\lambda_{w}}{\mu_{2 w}}
$$

such that $\Delta r_{w}=0$. For smaller jumps, $|\Delta \alpha|<\left|\Delta \alpha_{c}\right|$, the sign of $\Delta r_{w}$ is the one predicted by the linear approximation, but for larger jumps, $|\Delta \alpha|>\left|\Delta \alpha_{c}\right|$, the sign of $\Delta r_{w}$ is the opposite to the prediction of the linear approximation. For the sake of concreteness, in Fig. 4 we have repeated the numerical experiment of Fig. 2, but with larger intensity jumps. From the Monte Carlo simulation, we obtain the critical 


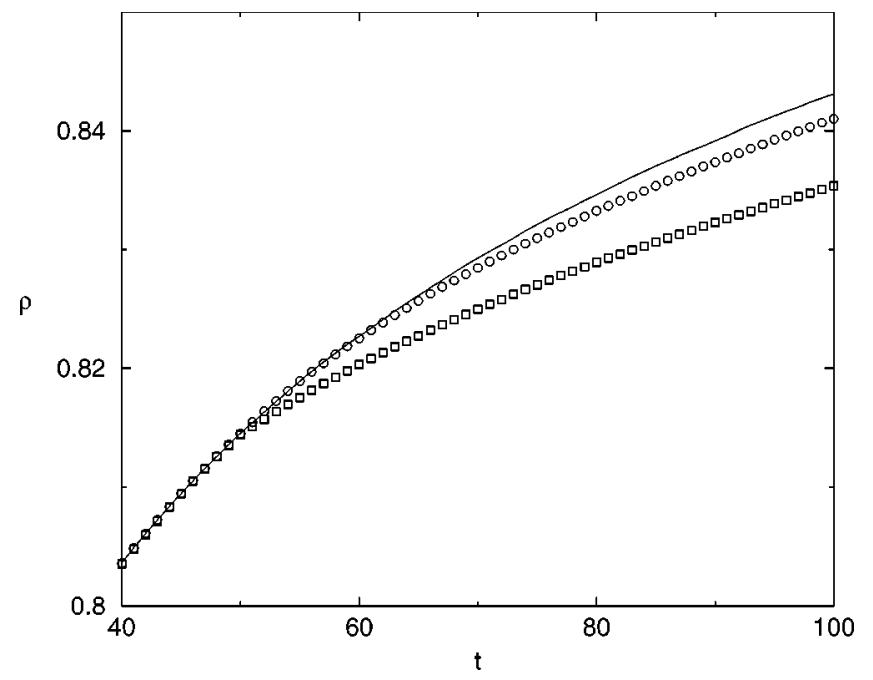

FIG. 4. Time evolution of the density $\rho$ when the vibration intensity $\alpha$ is changed at $t_{w}=50$, where $\lambda<0$, from $\alpha=0.15$ to $\alpha^{\prime}=0.05$ (circles) and 0.03 (squares), respectively. The curve corresponding to a constant vibration intensity $\alpha=0.15$ is plotted for reference (solid line). For such large jumps, the linear approximation is not valid, and the compaction rate does not increase. In fact, $\alpha^{\prime}=0.05$ corresponds to the critical value $\Delta \alpha_{c}$, for which no change in the compaction rate is observed for short times.

value $\Delta \alpha_{c} \simeq-0.1$ for $\alpha=0.15$ and $t_{w}=50$. Then, at $t_{w}=50$ we change the vibration intensity from $\alpha=0.15$ to $\alpha^{\prime}=\alpha$ $+\Delta \alpha_{c}=-0.05$, finding that the compaction rate does not change in the short-time limit $t-t_{w} \rightarrow 0$. Moreover, if the vibration intensity is further decreased, $\alpha^{\prime}=0.03$, the compaction rate also decreases, while the linear approximation predicted an increase of the compaction rate if $\alpha^{\prime}<\alpha$, since $\lambda_{w}<0$.

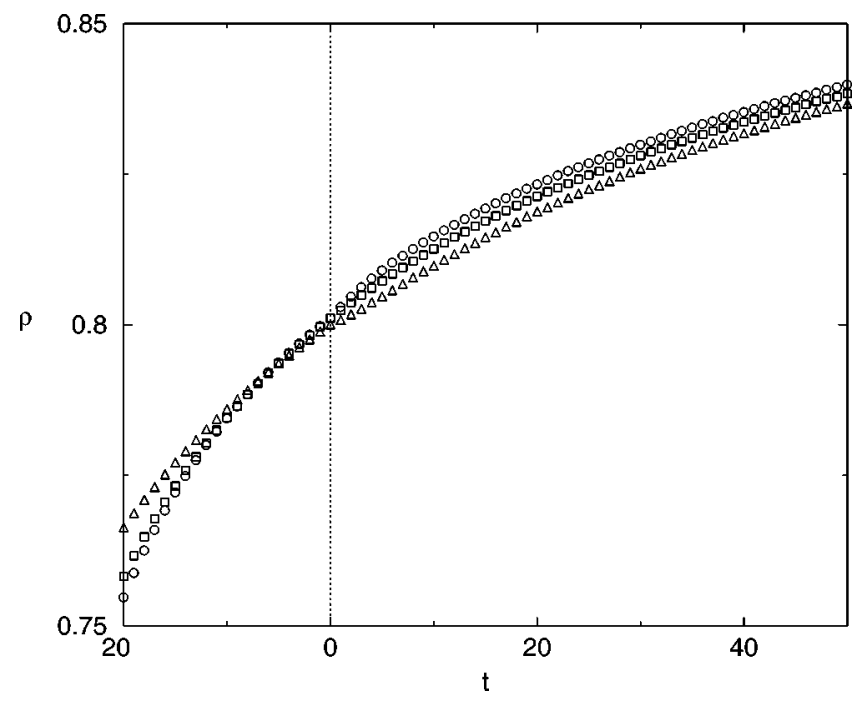

FIG. 5. Time evolution of the density for a system, which was tapped up to the same density $\rho=0.8$ using three different intensities, $\alpha=0.2$ (circles), 0.15 (squares), and 0.1 (triangles). Afterwards, the system was always tapped with $\alpha^{\prime}=0.15$. The time origin for each experiment has been taken at the time when the system reached the prescribed density, namely, $\rho=0.8$. The evolution for $t>0$ strongly depends on the prehistory of the system.
Following Ref. [4], we have also considered another series of numerical experiments where the system was tapped up to the same density with three different intensities, $\alpha$ $=0.2,0.15$, and 0.1 , respectively. Afterwards, the system was always tapped with the same intensity $\alpha^{\prime}=0.15$. The time evolution of the density is shown in Fig. 5, where the time origin for each experiment has been taken at the time when the system reached the prescribed density, namely, $\rho$ $=0.8$. The figure clearly shows that the evolution of the density for $t>0$ strongly depends on the previous tapping history, indicating the relevance of short-term memory effects. Mathematically, this is equivalent to say that $\mu_{1}(t)$ and $\mu_{2}(t)$ in Eq. (1) are not determined univocally by the density at the same time, so that it is not in fact a closed first-order ordinary differential equation. Note that in all the plotted curves the jump in the compaction rate has opposite sign than the variation of the intensity. We have verified that $\lambda(t)$ is negative at the time in which the intensity is modified in all cases, the behavior being then consistent with the theory.

\section{DISCUSSION}

Along this paper, we have studied the nonequilibrium linear response of a vibrated granular system to an instantaneous change in the intensity of the taps. In the first part, a general theory was developed on the basis of some plausible hypothesis about the mesoscopic dynamics of the system. The results are in qualitative agreement with the experimental observations. In particular, the presence of short-term memory effects appears as correlated with the relaxation properties of the system at constant intensity. An important theoretical prediction, not observed in the experiments yet, is the existence of a critical time $t_{c}$. For times $t<t_{c}$ the response of the system to a change in the intensity is "normal," in the sense that an increase in the intensity produces a positive jump in the compaction rate. On the other hand, for $t>t_{c}$, an "anomalous" response is produced. The change in the compaction rate has opposite sign than that of the modification of the vibration intensity, in contrast with the long-time behavior found in experiments, where the relaxation is faster for larger vibration intensity.

In the second part of the paper, a simple model for compaction has been considered. It is shown to fit perfectly into the general scheme developed before, allowing a detailed quantitative analysis of the theoretical predictions. This is not a peculiarity of this model, since the "parking" model [9] also verifies all the conditions assumed in the theoretical framework. In fact, this is not surprising because this latter model has a mathematical structure very similar to the one considered in this paper [6].

In Sec. IV we have shown that our model reproduces the experimentally observed behavior of the system when submitted to changes in the vibration intensity under different conditions [4]. Now we will refer to a more complicated pattern of changes in the intensity that are also discussed in Ref. [4]. First, the system is shaken with an intensity $\Gamma_{0}\left(\alpha_{0}\right.$ in the model notation) for a long period of time, so that the system practically reaches a steady density. Afterwards, at a time taken as $t=0$, the intensity is switched to $\Gamma>\Gamma_{0}$ for a 


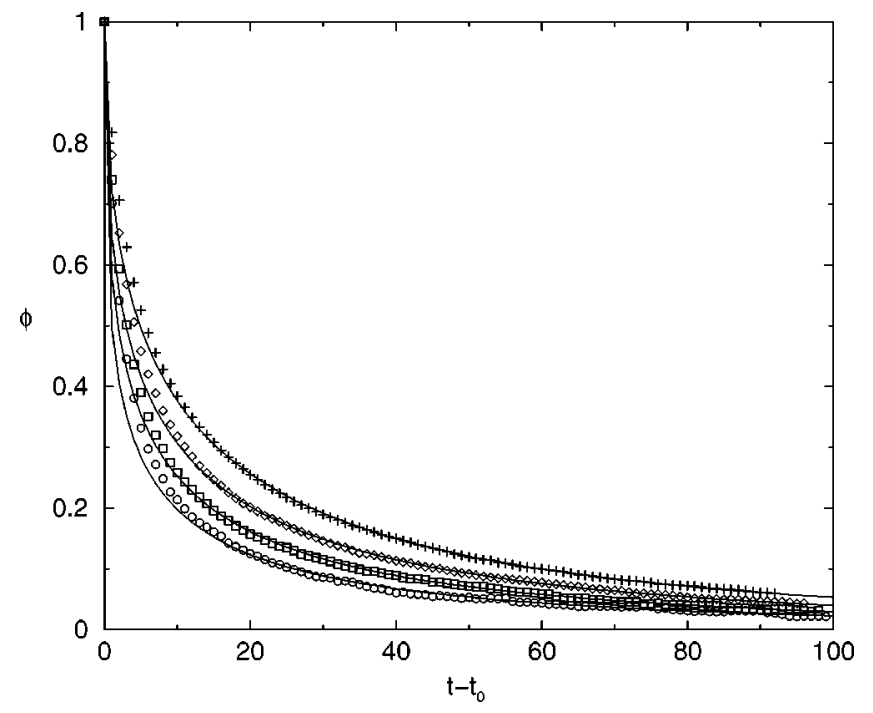

FIG. 6. Relaxation function $\phi(t)$, defined in Eq. (43), of the model, when it is prepared by tapping for a long time with $\alpha$ $=0.3$, and afterwards tapped for $t_{0}=1,2,4$, and 8 (from bottom to top) with a larger intensity $\alpha^{\prime}=0.5$. Finally, the intensity is turned back to the original intensity $\alpha=0.3$. All the curves tend to zero in the infinite time limit, and the solid lines are the best numerical fits to a KWW function.

given period of time $t_{0}$ and, finally, the system is tapped again with the original intensity $\Gamma_{0}$, and the subsequent relaxation of the system is studied. Experimentally it was found that the relaxation is slower the larger the $t_{0}$; the system "ages." Moreover, on the basis of a simple two-state model, it was proposed that

$$
\rho(t)-\rho_{s} \sim \frac{\exp \left(-\kappa_{0} t\right)}{t}
$$

for $t-t_{0} \gg 1$. In the above expression, $\kappa_{0}$ is a decreasing function of $t_{0}$. Josserand et al. [4] also reported that the relaxation can be fitted by a superposition of exponentials, all of them with the same amplitude. We have carried out numerically this kind of experiments in our model. In Fig. 6 we present the results obtained with $\alpha_{0}=0.3, \alpha=0.5$, and four different values of $t_{0}$, namely, $t_{0}=1,2,4$, and 8 from bottom to top. The plotted response function $\phi(t)$ is defined as

$$
\phi(t)=\frac{\rho_{s}\left(\alpha_{0}\right)-\rho(t)}{\rho_{s}\left(\alpha_{0}\right)-\rho\left(t_{0}\right)}
$$

where $\rho\left(t_{0}\right)$ is the density of the system at the time in which the the intensity is switched back to $\alpha_{0}$. For the model, the steady values of the density can be computed analytically [6], and it is $\rho_{s}(\alpha=0.3) \simeq 0.8371$. For a given time interval $t-t_{0}, \phi(t)$ increases with the "waiting" time $t_{0}$. Thus, the relaxation is slower for larger $t_{0}$, consistently with the experimental observation [4]. Also, we have fitted (solid lines) the data to a stretched exponential or KWW function [19],

$$
\rho_{K W W}(t)=\rho_{s}\left(\alpha_{0}\right)-\left[\left[\rho_{s}\left(\alpha_{0}\right)-\rho\left(t_{0}\right)\right] \exp \left[-\left(\frac{t-t_{0}}{\tau}\right)^{\beta}\right],\right.
$$

with $\tau$ and $\beta$ being fitting parameters. As observed in the figure, the fit is quite satisfactory, except for times very close to $t_{0}$ and, probably, for very large times. The parameter $\beta$ in Eq. (44) measures the width of the relaxation-time distribution. The values we have found go from $\beta=0.366$ for $t_{0}$ $=1$ to $\beta=0.478$ for $t_{0}=8$. The latter is close to the value $1 / 2$, characteristic of systems whose dynamics are dominated by one-dimensional diffusive processes. However, the KWW relaxation is not equivalent to a superposition of exponentials with the same amplitude, as proposed in Ref. [4]. Therefore, this point deserves more work in the future, both theoretically and experimentally. With respect to the long-time behavior predicted by Eq. (42), we could not reach a definite answer. Although the numerical data seems to be compatible with it, the noise is too large and further high-precision studies would be required.

Finally, a crucial point in the analysis presented in this paper is the small amplitude of the perturbation in the vibration intensity $\Delta \Gamma$. As pointed out at the end of Sec. III, the behavior following a large change in the intensity may be different. In the model considered in Sec. IV the nonlinear corrections are very simple, leading always to a decrease in the compaction rate and to the appearance of a critical value of the intensity jump, such that no change in the vibration intensity is observed in the short-time regime. Moreover, for jumps larger than the critical one, the sign of the change in the compaction rate is reversed as compared with the prediction of the linear approximation. We think that it is worth looking for this kind of behavior in other models for compaction, and also in experiments with real granular systems.

\section{ACKNOWLEDGMENTS}

This research has been partially supported by the Dirección General de Investigación Científica y Técnica (Spain) through Grant No. PB98-1124.
[1] J.B. Knight, C.G. Farndich, C.N. Lau, H.M. Jaeger, and S.R. Nagel, Phys. Rev. E 51, 3957 (1995).

[2] E.R. Nowak, J.B. Knight, E. Ben-Naim, H.M. Jaeger, and S.R. Nagel, Phys. Rev. E 57, 1971 (1998).

[3] M. Nicodemi, Phys. Rev. Lett. 82, 3734 (1999).

[4] C. Josserand, A. Tkachenko, D.M. Mueth, and H.M. Jaeger, Phys. Rev. Lett. 85, 3632 (2000).

[5] D.A. Head, Phys. Rev. E 62, 2439 (2000).
[6] J.J. Brey, A. Prados, and B. Sánchez-Rey, Phys. Rev. E 60, 5685 (1999).

[7] J.J. Brey, A. Prados, and B. Sánchez-Rey, Physica A 275, 310 (2000).

[8] P.L. Krapivsky and E. Ben-Naim, J. Chem. Phys. 100, 6778 (1994).

[9] E. Ben-Naim, J.B. Knight, E.R. Nowak, H.M. Jaeger, and S.R. Nagel, Physica D 123, 380 (1998). 
[10] A. Prados, J.J. Brey, and B. Sánchez-Rey, Physica A 284, 277 (2000).

[11] S.F. Edwards and R.B.S. Oakeshott, Physica A 157, 1080 (1989); A. Mehta and S.F. Edwards, ibid. 157, 1091 (1989).

[12] S.F. Edwards and D.V. Grinev, Phys. Rev. E 58, 4758 (1998).

[13] A. Barrat, J. Kurchan, V. Loreto, and M. Sellitto, Phys. Rev. Lett. 85, 5034 (2000); e-print cond-mat/0011492; S. Barrat and V. Loreto, J. Phys. A 336, 4401 (2000).

[14] A.J. Kolan, E.R. Nowak, and A.V. Tkachenko, Phys. Rev. E 59, 3094 (1999).

[15] J. Talbot, G. Tarjus, and P. Viot, Phys. Rev. E 61, 5429 (2000).
[16] J. Talbot, G. Tarjus, and P. Viot, e-print cond-mat/0008183.

[17] In the lower graph in Fig. 2 of Ref. [3], it is observed that for $t-t_{w} \rightarrow 0$, when the intensity of the vibration is increased, the height of the system decreases, i.e., the density increases. This graph corresponds to the lowest intensity considered, so that the waiting time $t_{w}$ is much smaller than the characteristic relaxation time. This explains the change in behavior as compared with the other two plots in the same figure.

[18] J. J. Brey and A. Prados (unpublished).

[19] G. Williams and D.C. Watts, Trans. Faraday Soc. 66, 80 (1970). 